\title{
Analytical methods for determination of trans-C18 fatty acid isomers in milk fat. A review
}

\author{
M. Ledoux ${ }^{1 *}$, L. Laloux ${ }^{2}$ and R. L. Wolff ${ }^{3}$ \\ ${ }^{1,2}$ Agence Française de Sécurité Sanitaire des Aliments (AFSSA), \\ Unité Qualité du Lait et Analyses Sensorielles, 10 rue Pierre Curie, 94704 Maisons Alfort Cedex, France \\ ${ }^{3}$ Université Bordeaux I, Institut des Sciences et Techniques des Aliments de Bordeaux (ISTAB), Laboratoire de \\ Lipochimie Alimentaire, avenue des Facultés, 33405 Talence, France
}

\begin{abstract}
This review describes analytical methods developed for the separation, identification and quantitation of trans-isomers of unsaturated fatty acids. Although these methods are applicable to a wide variety of food matrices, we have particularly emphasised the analysis of trans unsaturated fatty acids in milk fat. Various methods are described such as infrared spectroscopy, direct gas chromatography, gas chromatography in combination with either thin-layer chromatography or liquid chromatography both impregnated with silver nitrate and gas chromatography coupled with mass spectrometry. The field of application, the advantages and the drawbacks of each method are evaluated and discussed.
\end{abstract}

Key words. Trans fatty acid - milk product - chromatography - spectrometry - analytical method.

\section{Introduction}

Ethylenic bonds of unsaturated fatty acids can be either in cis configuration, a geometry where both hydrogen atoms are on the same side of the double bond, or in trans configuration, where the two hydrogen atoms are on opposite sides of the double bond. Furthermore, these double bonds can be located on different sides of the aliphatic chain, resulting in positional isomers. The physico-chemical, nutritional, biochemical and biological properties of the trans fatty acid isomers are different from those of cis isomers.

For the last three decades, trans fatty acids have been studied for their impact on human health and suspected to be harmful. Recent studies attempted to show a correlation between trans fatty acid ingestion and an increase of blood cholesterol level $[1,2,3]$ as well as a relationship between trans fatty acids and cardiovascular risks $[4,5,6,7]$. The conclusions of these epidemiological and experimental studies, debated in other respects, should be strongly supported by an in-depth knowledge of trans fatty acid levels in food and in the human diet. This knowledge requires appropriated analytical methods $[8,9,10]$.

Several analytical methods are reported for trans fatty acid determination and quantitation in food, specifically in milk fat. These analytical procedures mostly stem from separative techniques generally used for lipid analysis namely, gas chromatography (GC), thin layer chromatography impregnated with silver nitrate (Ag-TLC), reversed-phase liquid chromatography (LC) and liquid chromatography impregnated with silver nitrate (Ag-LC), and from detection methods commonly applied to lipid determination such as infrared spectroscopy (IR), Fourier Transformed infra-red (FTIR), flame ionisation detection (FID) and mass spectrometry (MS) [11]. Each of these methods exhibits advantages and drawbacks. Improvements in accuracy and effectiveness of results can be obtained by combining techniques, e.g. GC-MS with preliminary fractionation on Ag-TLC or Ag-LC, GC-FTIR, etc. Unfortunately, there is not one allencompassing method in this field. The use of one method over another depends on several factors such as the goal of the studies, the nature of samples, etc. [8,12,13]. Actually, the food manufacturer will be interested in the total level of trans fatty acids, whereas the biochemist or the nutritionist will want to measure the individual quantity of each isomer. Individual separation of each isomer allows for an optimal quantitation of trans fatty acids, preventing any risk of under- or over-estimation due to the method. Furthermore, the quantitation of each isomer is essential for certain studies, especially trans fatty acid levels in human tissues related to source, since the trans fatty acid composition varies when the diet includes milk products, margarine or oils subjected to heat treatment in production or when used in processing, such as frying $[10,12,14,15]$.

\section{Infrared spectroscopy (IR)}

Trans ethylenic bonds show a specific absorption in the infrared spectrum from 956 to $976 \mathrm{~cm}^{-1}$ with a maximum absorbance at $967 \mathrm{~cm}^{-1}$ [16]. This method is fast, easy to apply and useful for routine analysis [13,17,18,19,20,21,22]. 
However IR-spectroscopy is inappropriate to study individual trans fatty acid isomers since it does not give information on the ratio of the different isomers, or specific details on the type of isomers, such as chain length, number and position of trans bonds. Furthermore, results obtained using IR spectroscopy are higher, sometimes up to twice those obtained by chromatography [17,20,23,24]. For instance, Smith et al. [23] reported levels from 3.5 to $4.2 \mathrm{~g}$ trans fatty acids per $100 \mathrm{~g}$ total fatty acids in milk fat using IR spectroscopy and from 1.8 to $2.0 \mathrm{~g}$ per $100 \mathrm{~g}$ in the same samples using GC. However, repeatability and reproducibility are better using GC than using IR, especially when food products are low in trans fatty acid levels, which is the case for milk fat $[22,25]$.

Several reasons could explain these discrepancies. On one hand, triacylglycerols absorb in the infrared spectrum at about $970 \mathrm{~cm}^{-1}$ as well, which leads to an apparent increase in the trans fatty acid level measurements [17,20,22]. This problem can be circumvented by measuring trans fatty acid methyl ester absorption [17,22]. On the other hand, IR spectroscopy determines all trans ethylenic bonds, so multi-trans polyunsaturated fatty acids are measured as many times as double bonds are present. This may lead to an overestimation in terms of the percent of total fatty acids $[20,22,26]$. Moreover, the IR determination of total trans content of milk fatty acid methyl esters (FAME) was not fully satisfactory because the trans band overlapped with the conjugated diene band. IR accuracy was also limited by the fact that absorptivity of methyl elaidate, used as calibration standard, was different from those of the minor trans fatty acids found in milk [27].

So, great limitations in the use of IR to determine the trans content of milk and milk products were the lack of accuracy, especially for low level of trans fatty acids (case of milk fats), and the global measure of all-trans instead of individual determination of each trans fatty acid isomer.

Emergence of Fourier-transform infrared spectroscopy (FTIR) $[18,19,20]$ and the use of computer-assisted spectral subtraction procedures [21,22] allowed for the improved detection efficiency of this method. Unfortunately, results obtained by this new method yielded somewhat greater levels than chromatographic values, even if discrepancies were significantly reduced as a result of using an IR method. Furthermore, some large variations were noticed in FTIR measurements of edible fats that contained low levels of trans fatty acids (less than $15 \%$ of total fatty acids); this is usually the case with milk products $[8,22,26]$.

A method combining GC-FTIR has been used recently to determine ethylenic bond geometry [28]. The advantage of this combined method is to directly obtain the IR spectra of each fatty acid methyl ester (FAME) peak after chromatography [29]. This method is still very recent, very expensive, not commonly used and requires technical expertise. At the moment, GC-FTIR has not been used extensively in various matrices, since little information is available about analytical performance. Such analytical methodology still remains a «high-tech» procedure for research institutes and seems not very well adapted to routine control laboratories involved in food composition analysis.

\section{Direct gas chromatography}

The major advances of this method in regards to trans fatty acid chromatographic analysis are the use of capillary columns substituted for packed columns, emergence of new more polar phases, and increased length of the capillary columns. It should be noted that GC column efficiency is proportional to the square root of column length and the resolution is strongly influenced by the selectivity, therefore increasing column length will lead to appreciably higher resolution, and selectivity modification will affect significantly separation as well $[12,30]$. For instance, during the 1970's, the use of polar packed columns (such as SP-2340 or OV275) did not afford separation of cis-18:1 fatty acids from trans-18:1 isomers, and even poorer quantitation of each of the positional isomers [13,23]. Emergence of capillary columns bonded with the same phases allowed for a slight improvement of separation, though still not suitable. These capillary columns gave essentially an interesting reduction of width peaks $[12,31]$. However, increasing the length of the column (SP-2340 or FFAP) did not seem to improve separations [31,32].

Recently available polar columns, bonded with cyanoalkyl polysiloxan phase, such as SP-2560 [33,34], CPSil 88 [4,8,35,36] or BPX-70 [29], demonstrated significant improvement of both trans-18:1/cis-18:1 separation and quantitation of individual trans-18:1 isomers. Increasing the length of these columns from $50 \mathrm{~m}$ to $100 \mathrm{~m}$ results in a significant improvement in the separation of the different geometrical and positional isomers [4,8,26,37]. Using these phases, trans-18:1 positional isomers were eluted in the order of the double bond progression along the carbon chain from carboxyl $(\Delta 5-\Delta 6, \Delta 7, \Delta 8 \ldots)$; trans isomers up to $\Delta 11$, or $\Delta 13-14$ (depending on column type), have retention times lower than that of oleic acid (cis-9). A first group of 18:1trans fatty acids, including trans-6-8, trans-9, trans-10, trans-11, appeared relatively well isolated before the peak corresponding to oleic acid (18:1 cis-9). The trans-6-8 isomers were not resolved. A second group, including trans-12 through trans-15, eluted with oleic acid and other cis-18:1 isomers (cis-6 through cis-11) [4,8,13]. Under optimal operating conditions, the Precht and Molkentin's group [12] partially resolved the 18:1 trans-15 peak from the 18:1 cis-9. Our attempts to optimize 18:1 chromatographic separation led to overlapping of trans-12 through trans-14 with the cis group and to a shouldering of the trans-15 just after the main cis-9 peak (Fig. 1a) [38].

Using 100-m SP-2380 capillary column held at $242{ }^{\circ} \mathrm{C}$ (isotherm condition), Thompson [34] obtained two different 18:1 fatty acid groups each eluted in its own peak, trans group on one hand, cis isomers on the other.

Gas chromatography using these very polar phases resulted in suitable resolution for linoleic acid geometrical 


\section{Review}
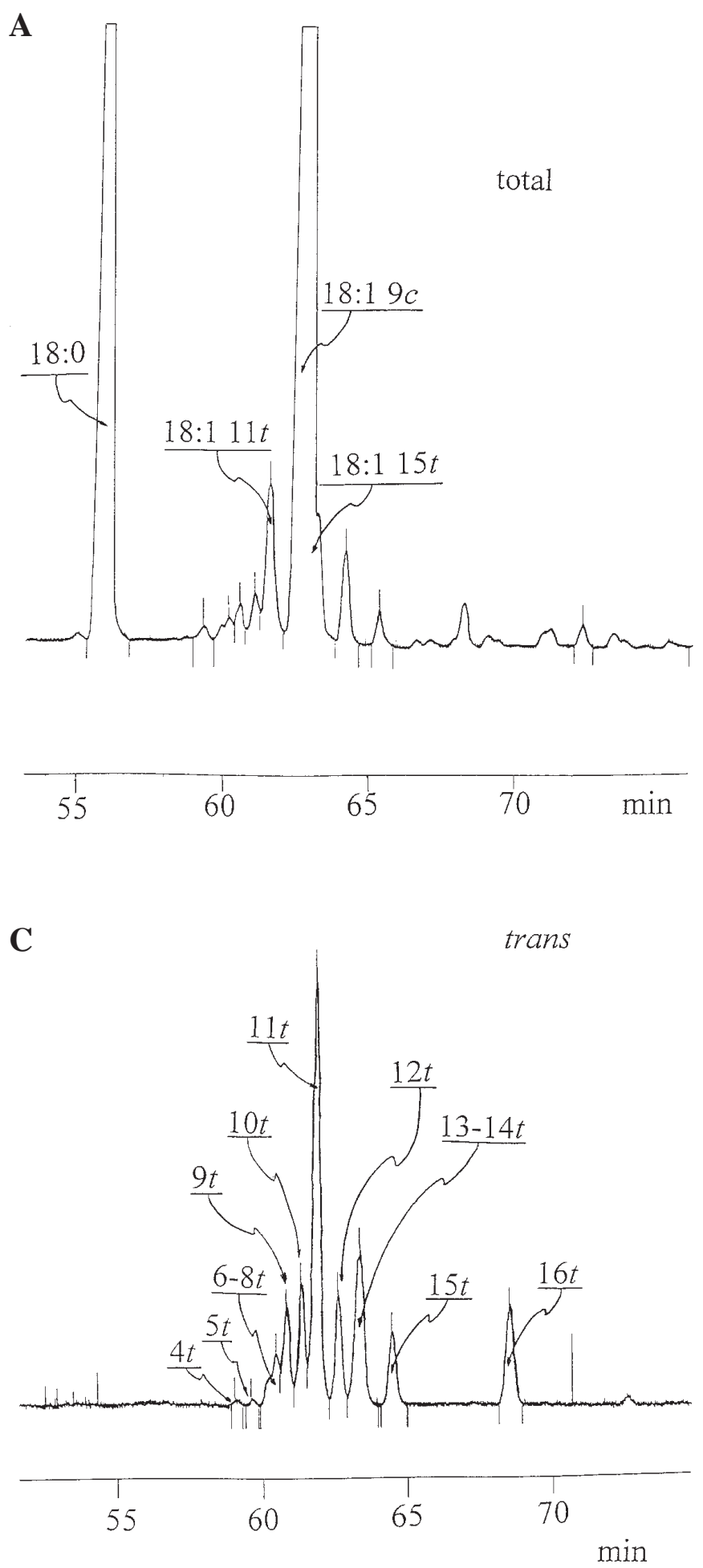

isomers; Berdeaux et al. [29] observed individual separation of linoleic acid 18:2 cis-9, cis-12 and of its 3 geometrical isomers trans-9/trans-12, cis-9/trans-12 and trans-9/cis-12 using a 30-m BPX70 capillary column. This analysis becomes more complicated when mono-trans-18:2 or

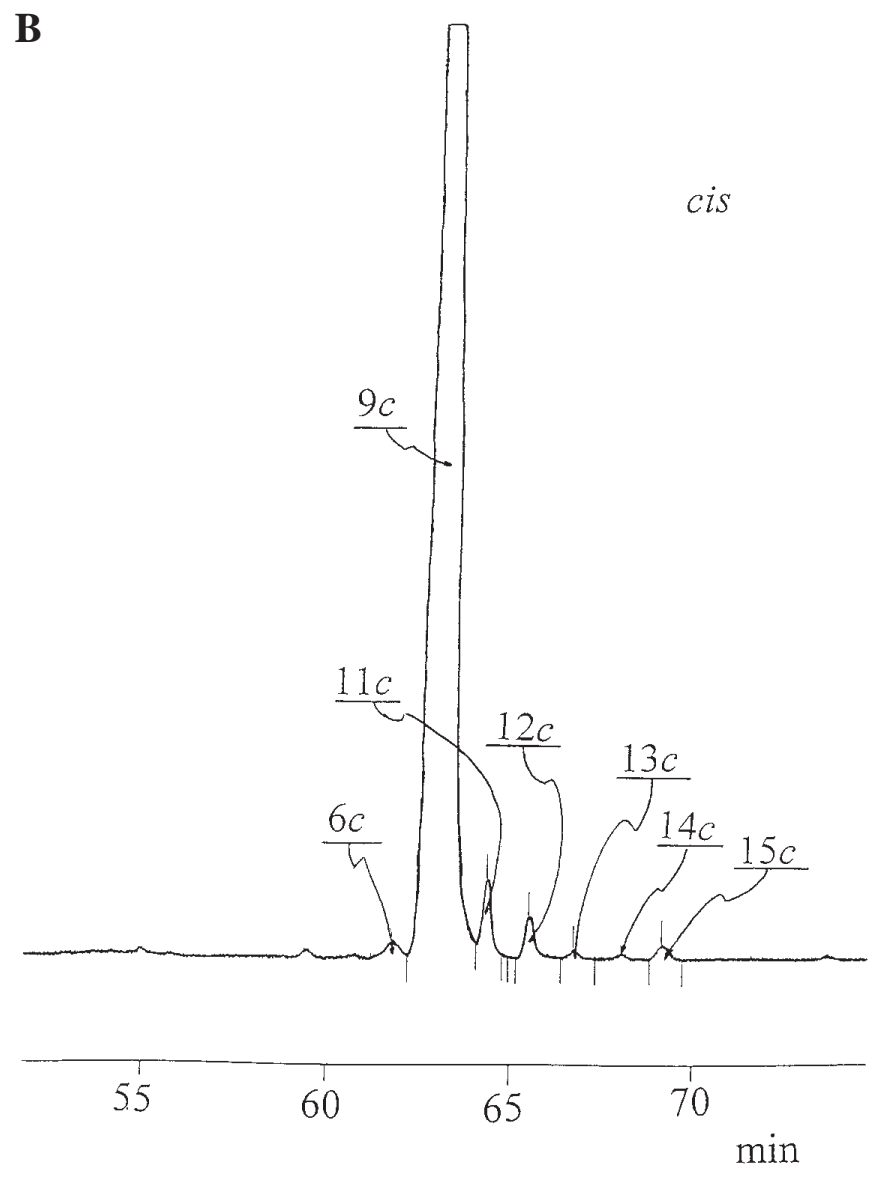

Figure 1. Partial chromatograms of fatty acid isopropylic esters (FAIPE) prepared from goat milk fat, analyzed on a 100-m capillary column CP-Sil 88 (Chrompack), $160^{\circ} \mathrm{C}, 220 \mathrm{KPa}$ (helium). $\mathrm{A}=$ FAIPE before fractionation with Ag-TLC. $\mathrm{B}=$ cis-monoenoic acid fraction isolated by $\mathrm{Ag}$-TLC $(\mathrm{x} 4) . \mathrm{C}=$ trans-monoenoic acid fraction isolated by Ag-TLC (x8) from M. Ledoux (1999) [38].

di-trans-18:2 positional isomers are present, even when using a 100-m column. Numerous peaks were poorly resolved, or eluted together in one peak, or co-eluted with cis-18:1 isomers [10,33]. This problem was all the thornier when conjugated linoleic acids (CLAs) were in relatively 
high proportions, as in milk products $[35,39]$. CLAs, polyunsaturated fatty acids exhibiting anticarcinogenic properties, have retention times closed to 20:0 and 20:1 fatty acid peaks [29]. Using a 100-m CP-Sil 88 in a gradient program mode, CLA compounds were isolated in 3 groups: trans/cis, cis/cis and trans/trans isomers (in the elution order) [40]. In this study, the CLA region of FAME direct GC trace for cheese revealed a complex mixture of 10 peaks attributed to 19 or 20 isomers identified subsequently using Ag-LC fractionation prior to GC-MS.

An International Standardization Organization (ISO) guideline regarding trans fatty acid determination in animal and vegetable edible fats using GC is under study at the moment [41]. However, even if peak co-elutions are reduced using optimal phases in columns as long as $100-\mathrm{m}$, all peaks are not perfectly resolved. This fact was a great limitation of direct GC for studies which needed individual identification and quantitation of each isomer but it may provide relatively quickly a good idea of the C18 unsaturated fatty acid content and a rough isomer distribution. In order to obtain better separation with the same kind of columns, a pre-fractionation of cis/trans geometrical isomers is needed by argentation (silver salts) liquid chromatography.

\section{Argentation thin layer chromatography (Ag-TLC)}

Geometric isomer separation using thin layer chromatography plates impregnated with silver nitrate, is based on the property of trans isomers to form unstable components when reacted with silver salts. These components are different than those formed with cis isomers. This principle was applied for the first time by Nichols in 1952 [42]. The retention front (Rf) during migration on thin layer plates depended mainly on geometric conformation of ethylenic bonds, as well as on the degree of unsaturation and position of double bonds on carbon chain [12]. A good adaptation of eluting conditions (such as choice of solvent) allowed for distinct spots as a function of unsaturation. In this way, monoenoic, dienoic and polyenoic acids can be more or less separated and individually quantitated $[23,39,43]$. Other considerations which could optimize trans/cis Ag-TLC separations are methods of $\mathrm{Ag}$ ion impregnation on the silica gel, $\mathrm{Ag}$ ion impregnation rate, nature of the counter-ion, activation of plates, tank saturation or not, and elution temperature. Some practical considerations about silver-ion chromatography of lipids were reviewed by NikolovaDamyanova [44]. In most cases, the plates were dipped in a $5-20 \%$ (wt/vol.) $\mathrm{AgNO}_{3}$ solution in acetonitrile or water, then dried and activated for one hour at $100-110{ }^{\circ} \mathrm{C}$ and developed in saturated tanks at room temperature in hexane - diethyl ether or petroleum ether - diethyl ether $[4,23,30,39,45]$.

In operating conditions for monoenoic trans fatty acid analysis, Ag-TLC of a fatty acid methyl ester extract led to the formation of four main spots corresponding to polyunsaturated fatty acids, cis-monoenoic fatty acids, trans- monoenoic fatty acids, and saturated fatty acids (in increasing $\mathrm{Rf}$ order). Aro et al. [4] noticed intermediate spots as well, including mixtures of some isomers. According to these authors, the main spots contained $85 \%$ of total 18:1 fatty acids; extracting the intermediate spots allowed for improved extraction recovery up to $95.6 \%$, but these observations were not confirmed by any other research group. Wolff et al. [13,37] reported $100 \%$ recovery yield when operating conditions are fully optimised and controlled.

After Ag-TLC elution, Wolff $[43,46]$ observed 18:2 and 18:3 geometrical isomer fractions corresponding to the degree of trans unsaturation, in an increasing order: first trans,trans-18:2 then trans, cis- and cis,trans-18:2 then ditrans-18:3, then mono-trans-18:3 and finally linolenic acid (tri-cis-18:3). Ratnayake and Pelletier [47] observed less homogenous groups, however the initial oil mixture was more complex and included geometric and positional linoleic acid isomers.

At the end of thin-layer chromatographic runs, silica gel was scrapped off and FAME were extracted using methanol/sodium chloride/hexane [30] or diethyl ether [39] and then analyzed by GC. Recently, glass plates were replaced by plastic sheets that can be cut using scissors [22]. Silica gel is no longer scrapped from the sheet, but specific areas can be cut from the sheet and plunged into solvent solution for trans fatty acid extraction.

Overall, and despite few differences in published protocols (such as impregnation of thin layer plates, choice of developing and extracting solvents, etc.), Ag-TLC fractionation is the cheapest method and the easiest to use, since it requires only typical laboratory equipment [48]. But this methodology appeared to be time-consuming and laborious for some authors [4] and there is no possibility of automation.

\section{Argentation high-performance liquid chromatography (Ag-HPLC)}

Several attempts were conducted to adapt Ag-TLC to highperformance liquid chromatography (HPLC), the main problem being to obtain a stable and reproducible stationary phase with a controlled silver ion content and a reasonable working life $[20,44,49,50]$. Some practical considerations about Ag-HPLC of lipids were reviewed by NikolovaDamyanova [44]. Columns packed in the laboratory with silica gel impregnated with silver nitrate was the first technique used in Ag-HPLC scope, but preparation of impregnated columns in the lab required much practice and skill $[44,51]$. The main drawback of these columns was the $\mathrm{AgNO}_{3}$ bleeding while in use, which limited their working life. Silver ion are held much more strongly by the support when they are bound to a cation exchanger. Argentation techniques using ion exchangers gave more reproducible $\mathrm{AgNO}_{3}$ impregnation rates and such manufactured columns were more stable for a long period of time [52]. Silver ions can be added to mobile phases in reversed phase HPLC. In 
Figure 2. Ag-HPLC profiles of cheese (A) and cow milk (B) using three silverion columns in series from Sehat et al. (1999) [53].

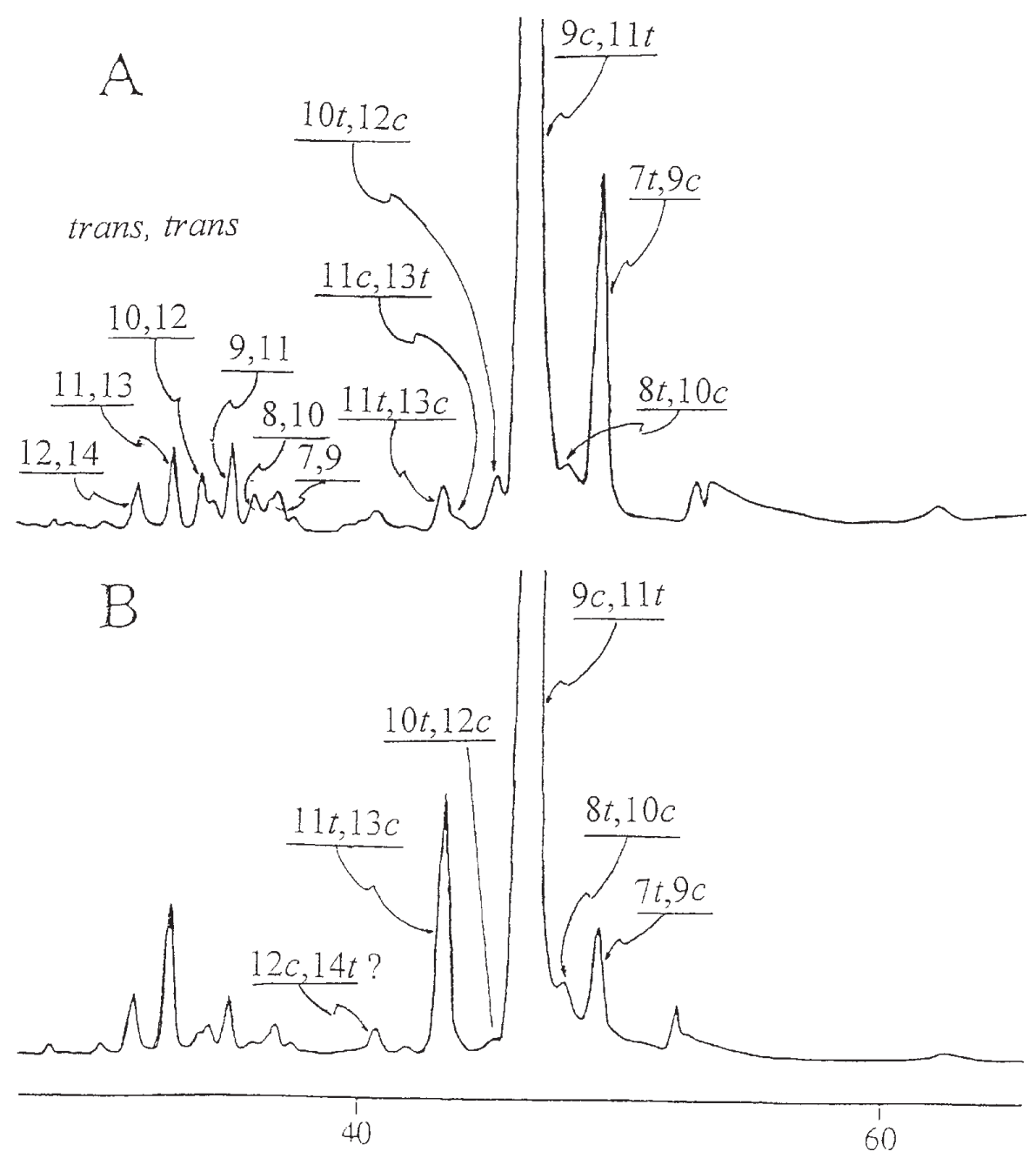

rated by Ag-HPLC into 16 peaks attributed to 19 CLA isomers which were identify using direct GC of FAME on 100-m polar capillary column, and GC-MS and GC-FTIR after Ag-HPLC fractionation and subsequent derivatization to DMOX derivatives. Sehat et al. [54] reported improved resolution of the CLA isomers by using two to six Ag-HPLC columns in series. In cow milk and cheese extracts, the minor trans-8, cis-10 18:2 isomer was resolved from the more abundant trans-7, cis-9 18:2 and cis-9, trans-11 18:2 acids, and the trans-10, cis-12 18:2 was separated from the major cis-9, trans-11 "rumenic" acid using three Ag-HPLC columns in series (Fig. 2). The authors claimed that the use of three Ag-HPLC columns was the best compromise to achieve, in a timely manner, resolution of most CLA isomers in biological matrices. But, as such columns are expensive and have a limited life time, this method is not practical for routine analysis laboratories.

Juanéda and Sébédio [54] reported the use of Ag-HPLC for separation, purification and quantitation of the eight cis/trans geometrical isomers of linolenic acid. These isomers were analyzed as phenacyclic esters. The liquid 
chromatography was performed on a silver nitrate bound Nucleosyl 5SA column, held at $10{ }^{\circ} \mathrm{C}$ and run in elution gradient mode using dichloromethane and methanol. In such conditions, the 8 isomers appeared individually.

\section{Trans isomer individual quantitation}

Individual quantitation of each trans monoenes fatty acid isomer, in an edible fat extract by chromatographic analysis, actually requires a 3 -step procedure, specifically cis/trans isomer separation by Ag-chromatography, trans monoenes fatty acid quantitation using GC, and total fatty acid ester quantitation in order to report trans monoenes fatty acid content as a percent of total fatty acids. Accuracy of results depends on trans monoenes fatty acid separation and quantitation effectiveness, as well as on the total fatty acid measurement efficiency. In the two analysis (trans FA and total FA), the use of suitable columns and appropriate operating conditions are essential. This was not always the case in published papers. Wolff $[13,36]$ drew attention to three main types of problems that frequently occur in total fatty acid analysis. First, non-quantitative esterifications and volatile fatty acid loss during evaporation steps can be a source of erroneous results in ruminant fat quantitation. Next, high initial temperatures in gradient programs induced solvent front and short chain fatty acid peak overlapping. This fact is the main cause of error in milk fat analysis. Lastly, the concept of theoretical response factors is not directly applicable to methyl esters of short-chain fatty acids, since their carbon deficiency is larger than expected from theory. Substituting the methyl group by an ethyl, propyl, or butyl group improved the flame-ionization efficiency of fatty acid esters gradually [55]. So, when FAME were used, results needed to be corrected using a correction factor for trans monoenes fatty acid determination as well as total fatty acid quantitation. In order to minimize some of these drawbacks, the use of fatty acid isopropylic esters (FAIPE) was recommended $[30,36]$. Wolff [36] reported that there was no need to apply correction factors to transform peak area percentages into fatty acid weight percentages when butyl or propyl esters were used. And he demonstrated that esters of $\mathrm{C}_{3}$-alcohols have conversion factors close to 1 , independently of the chain length of the acids. Christie [56] reviewed the use of isopropyl esters instead methyl esters for milk fat analysis. Short chain FAIPE were easily separated from the solvent front and these esters were less volatile than FAME (less evaporation loss).

Individual quantitation of polyunsaturated fatty acids (PUFA) with trans bound(s) answers nearly to the same rules. But cautions must be taken for transesterification of conjugated octadecadienoic acids. All acid-catalyzed procedures resulted in decreased cis/trans and increased trans/trans conjugated dienes [27]. Such methylation procedures resulted in loss of total CLAs and low recovery in cis-9, trans-11 18:2 "rumenic" acid. The alkaline-catalyzed procedures gave no isomerisation of CLAs, but they did not transesterify sphingolipids, and sodium methoxyde did not methylate free fatty acids [27]. The best compromised for milk fatty acids would be obtained with sodium methoxyde $\left(\mathrm{NaOCH}_{3}\right)$ followed by hydrochloride acid or bismuth fluoride $\left(\mathrm{BF}_{3}\right)$, or diazomethane followed by $\mathrm{NaOCH}_{3}$ [27]. This is a very important fact since milk and milk products are relatively rich in CLAs, fatty acids with anticarcinogenic properties.

Other kinds of derivatization were recommended for mass spectrometry of fatty acids; this point will be discussed later in the paragraph devoted to this technology.

\section{Ag-TLC coupled with GC}

GC coupled with a pre-separation by Ag-TLC, first applied to GC methods using 50-m highly polar columns (such as CP Sil-88, Chrompack), led to better results than using direct GC (without Ag fractionation) [22,35,36]. Associating the two chromatographic techniques allowed for separation of trans-18:1 fatty acid isomers as 6 or 7 peaks [8]. Later, Wolff and Bayard [30], and Molkentin and Precht [57] succeeded simultaneously in the quasi individual separation of almost all trans-18:1 isomers, using a 100-m CP Sil-88 column after a pre-separation by Ag-TLC. They noted up to 10 peaks for trans-18:1 fatty acids and 9 peaks for cis-18:1 isomers. In similar operating conditions, we obtained 10 peaks for trans-18:1 fatty acids and 7 peaks for cis isomers [38] (Fig. 1b et c). Using Ag-TLC fractionation prior to GC on a highly polar $100 \mathrm{~m}$ capillary column, 8 trans-18:1 isomers can be quantitated individually, but separation remained incomplete since 18:1 trans-6 to trans- 8 are eluted in one group and the 18:1 trans-13 and trans-14 isomers are not resolved [30,37,38,57]. Figure 3 showed an optimised separation of trans-18:1 fatty acid isomers using Wolff's recommendations [38]. Partial separation of the 18:1 trans-13 and trans-14 isomers can be realised by decreasing column temperature and increasing gas vector pressure. This adaptation, however led to a longer run time, which would not be suitable for a routine process [37].

Comparing direct $100-\mathrm{m}$ GC versus $100-\mathrm{m}$ GC with a pre-fractionation on Ag-TLC demonstrated that direct GC can under-estimate trans fatty acid content up to 15-45\% $[13,33]$. In a study on 198 human milk fat samples, Chen et al. [33] reported that an average of $20.8 \%$ of total trans18:1 fatty acids were actually quantitated as cis-18:1 fatty acid fraction due to peak overlapping. This study showed extreme deviations ranging from 9 to $30 \%$ as a primarily function of total trans-18:1 fatty acid content. Wolff et al. [13] evaluated the direct GC under-estimations by analysing 2340 food samples and 310 human tissue samples. These authors reported that under-estimations can reach up to $43 \%$. So, in order to compare all available data, results obtained using direct GC would need to be corrected.

Using FAIPE instead of FAME did not modify the trans18:1 isomer separation when using a combination of $\mathrm{Ag}$ TLC and GC, but additional overlaps occurred in direct GC [30]. Increasing the length of the columns from $50 \mathrm{~m}$ to $100 \mathrm{~m}$ results in a significant improvement in the separation 


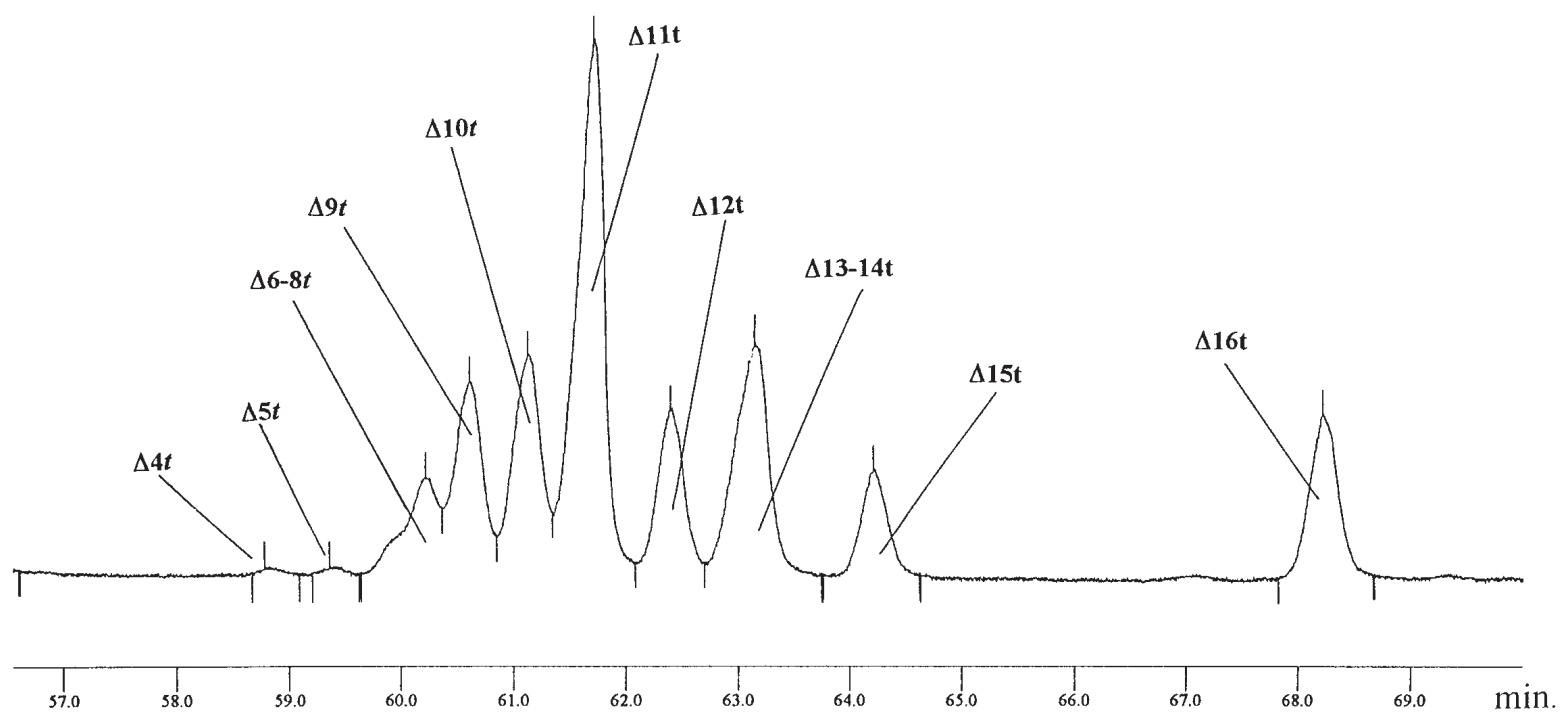

Figure 3. Chromatogram of trans-18:1 isomers as FAIPE prepared from goat milk fat and isolated by Ag-TLC (100-m capillary CP-Sil $88,160{ }^{\circ} \mathrm{C}, 220 \mathrm{KPa}$ (helium) from M. Ledoux (1999) [38].

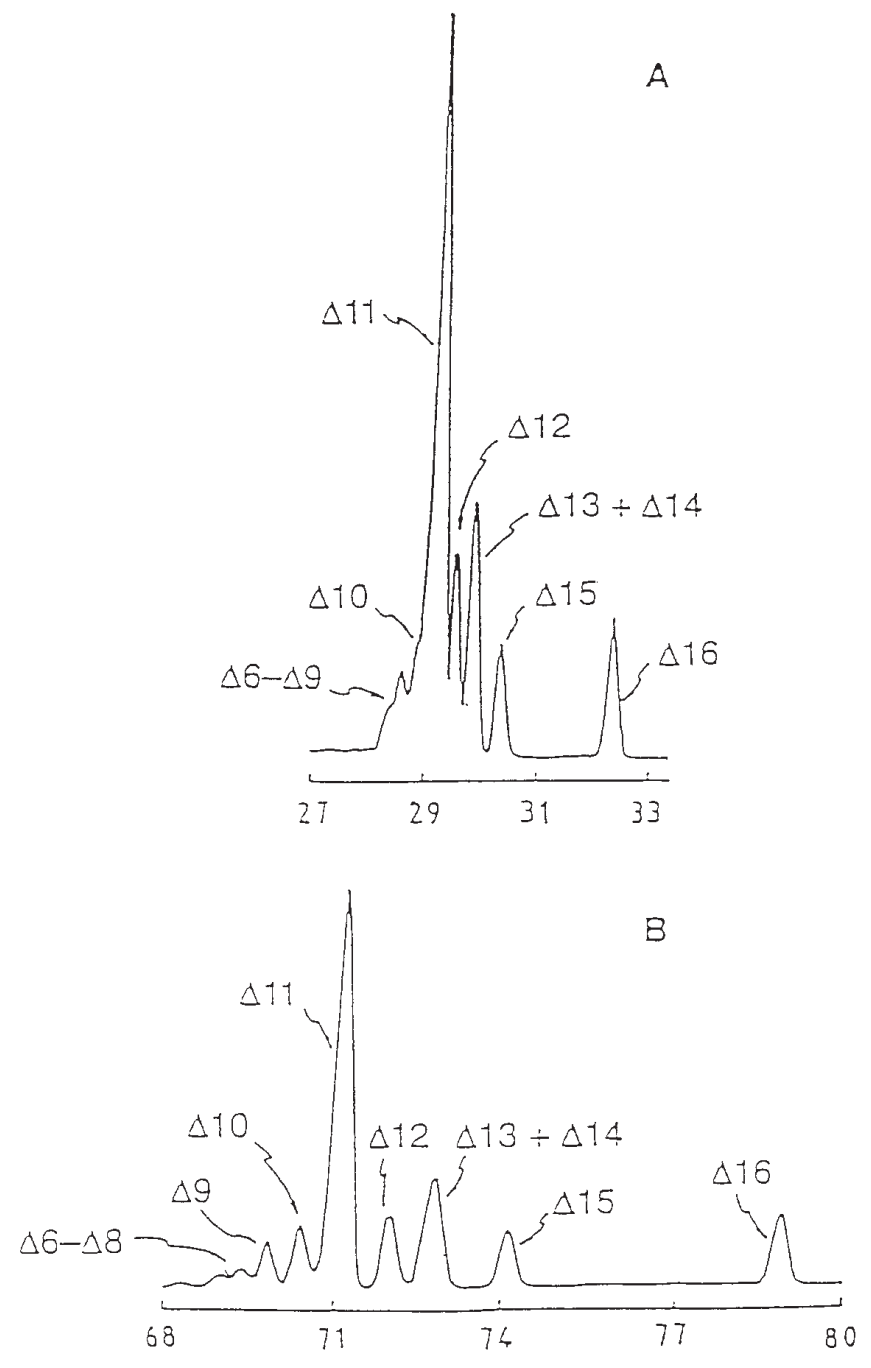

of the trans-18:1 fatty acid isomers after Ag-LC fractionation (Fig. 4) [30].

Silver nitrate thin-layer chromatography did not improve the separation of linoleic and linolenic geometric isomers. All isomers are individualized except cis-9,trans-12,trans-15 18:3 and trans-9,cis-12,trans-15 18:3 isomers that co-elute in the same peak using direct GC and Ag-TLC/GC combination as well (Fig. 5) [46]. For positional isomers (such as CLAs), separation was greatly improved using a combination of gas chromatography and argentation thin-layer chromatography, but at the cost of a corresponding increase of the run time [39]. In the same way, the pre-fractionation of FAME is useful to quantitate $\gamma$-linoleic acid (cis-6, cis-9, cis12 18:3) and to identify its geometric isomers [58].

GC combined with Ag-TLC allowed the individual separation of almost all trans monoenes and ameliorate the separation of trans-PUFA. This methodology was time-consuming and no automation was reported, so this procedure required considerable manipulations. These were drawbacks and limitations of the method. But Ag-TLC coupled with $\mathrm{GC}$ required only common laboratory equipment (except the $100 \mathrm{~m}$ highly polar capillary column) and were easy to use.

\section{Ag-HPLC coupled with GC}

GC combined with a pre-fractionation on Ag-HPLC seemed to be a good alternative to Ag-TLC prior GC. The HPLC was faster and less time-consuming than TLC and automa-

Figure 4. Chromatograms of the trans-18:1 fatty acids isolated from butterfat and analyzed as FAIPE either on a $50 \mathrm{~m} \mathrm{CP-Sil} 88$ (A) or on a 100-m CP-Sil 88 (B) capillary column from Wolff and Bayard (1995) [30]. 


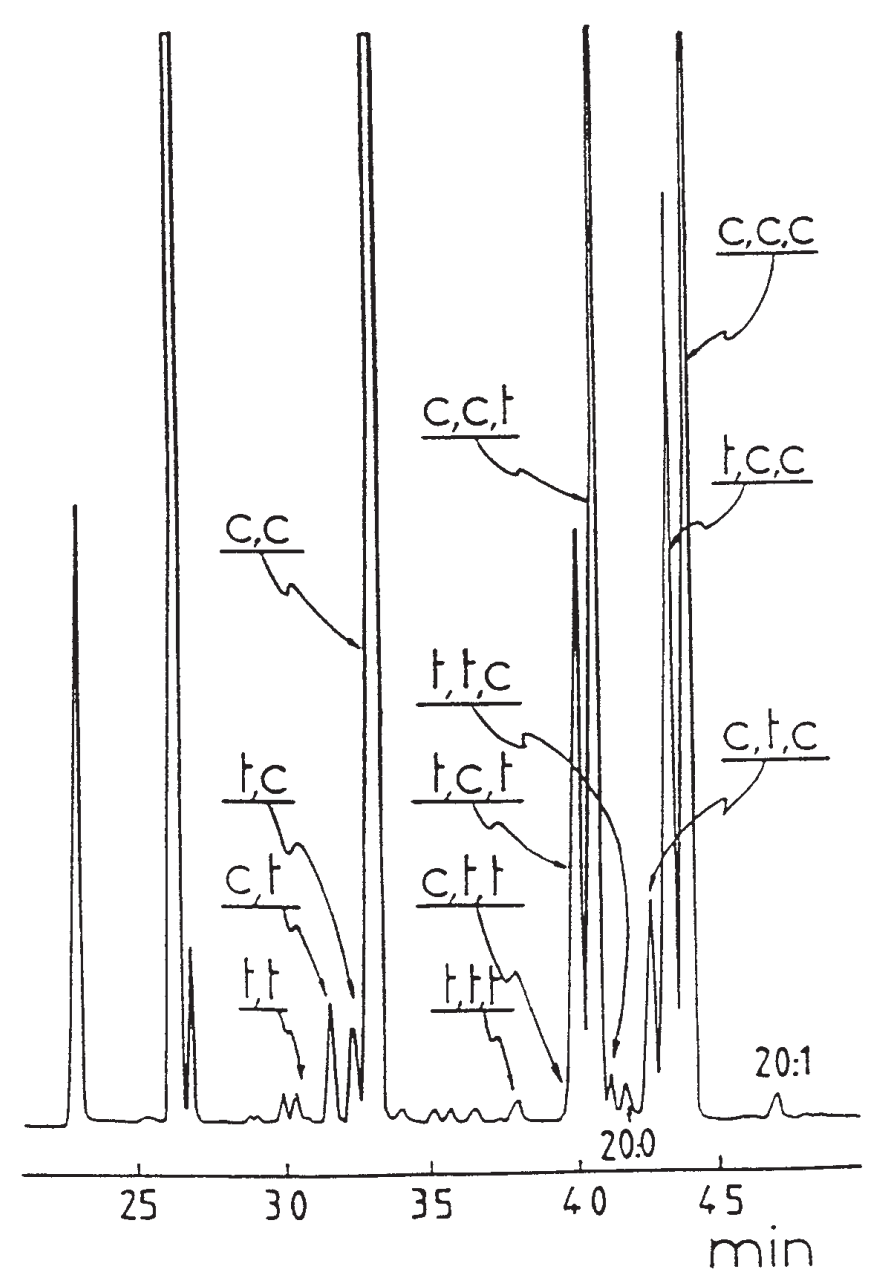

Figure 5. Partial chromatogram of FAME prepared from heated linseed oil. The configuration of double bonds are given in the order 9 and 12 for 18:2 n-6 acid geometrical isomers, and in the order 9,12 , and 15 for 18:3n-3 acid geometrical isomers from Wolff (1995) [46].

tion is possible using automated fraction collectors. The problem of unstable Ag-HPLC columns is on the way to being circumvented since commercially produced, consistent reproducible Ag-HPLC columns are becoming available.

The complete base-line separation of cis- and trans-18:1 isomers in two distinct groups makes the Ag-HPLC technique a good way to fractionate trans fatty acid prior GC analysis. This methodology has given good results with hydrogenated vegetable oils [20,49], but adaptation to milk matrices needs more investigation. In the same way, efforts should be made to adapt this technology to the trans-16:1 fractionation.

Sehat et al. [40] reported the identification of conjugated linoleic acid isomers in cheese by silver ion high performance liquid chromatography and gas chromatography combined with electron ionization mass spectrometry. Total CLA fatty acid methyl ester content ( $\%$ of total FAME) in extracted lipids was determined by direct GC and CLA isomeric composition (\% of total CLA FAME) measured by Ag-HPLC.

Ag-HPLC fractionation of cis/trans isomers will probably replace the Ag-TLC in the future in many laboratories involved in this field of research.

\section{Mathematical models}

Several authors proposed correction factors or mathematical equations to transform partial results obtained with direct $\mathrm{GC}$ into results equivalent to those obtained using $\mathrm{Ag}$ TLC/GC combination [35,57].

In the case of milk fat, the direct GC method underestimated the true trans-18:1 fatty acid content as determined by the combined method (Ag-TLC/GC) with a constant error of $0.367( \pm 0.162) \mathrm{g} / 100 \mathrm{~g}$ fat and a relative error of 8.3 $( \pm 2.6) \%$ [35]. From 61 butter chromatographic quantitations (direct GC and Ag-TLC/GC combination) and a linear regression analysis, Molkentin and Precht [57] presented a more precise equation :

total trans $-18: 1$ acids $=1.20911 \times$ trans $-6-11+0.927791$, with $r=0.9929$ and a standard error of estimation of $0.17 \%$.

Using this equation, only the $18: 1$ trans-6-11 group is quantified by direct $\mathrm{GC}$ on a $50-\mathrm{m}$ column. This equation was further improved and adapted for use with 100-m columns when one hundred butters from different areas in Germany and different period of the year were analysed:

$$
\begin{aligned}
& \text { total trans- } 18: 1 \text { acids }=1.05676( \pm 0.00381) \\
& \times \text { trans }[2]_{\mathrm{GC}}-0.001876( \pm 0.000514) \times 16: 0,
\end{aligned}
$$

with $r=0.99988(n=100)$ and a standard error of estimation of $0.065 \%$ [26].

The term "trans $[2]_{\mathrm{GC}}$ " in the equation corresponds to the sum of trans-4-14 with cis-6-8 contents as well as trans-16 overlapped cis-14 contents. The term "16:0" represents the content of palmitic acid (16) in the extract.

The same authors $[26,59]$ studied the correlation between triacylglycerol level and trans-18:1 acid content. From many chromatographic measures, and statistical and mathematical analysis (multiple regression analysis), the following formula was developed:

Total trans-18: 1 content $=3.7190 \mathrm{C} 26+2.4439 \mathrm{C} 28-$ $8.1505 \mathrm{C} 30+3.5187 \mathrm{C} 32-0.4819 \mathrm{C} 34-0.3737 \mathrm{C} 36+$ $0.3514 \mathrm{C} 40+1.3285 \mathrm{C} 42-1.342 \mathrm{C} 44-0.2689 \mathrm{C} 46+$ 0.9557 C48 - 0.7356 C52 + 1.0775 C54,

with $r=0.9977, n=100$.

$\mathrm{Cn}$ terms correspond to triacylglycerol contents obtained using GC for triacylglycerol with $n$ carbons. So, according to these authors, trans-18:1 acid content in bovine milk fat may be deduced from only triacylglycerol analysis, 
avoiding the cumbersome pre-fractionation of FAME combined with capillary GC.

The main limitation of mathematical models was that these methods can be applied only to milk fat, from bovine species, and only for trans-18:1 fatty acid isomers. The determination of trans positional isomers of octadecenoic acid in milk fat using Precht and Molkentin's triacylglycerol formula is limited to milk fats from bulk milk of a herd; triacylglycerol analysis of milk fat from single resulted in the incorrect determination of trans-18:1 [26].

\section{Mass spectrometry}

The goal of unsaturated fatty acid structural analysis was to determine the position of double bonds in the carbon chain and their configurations, either trans or cis [29,60]. Electronic impact mass spectrometry of fatty acid methyl esters led to $\pi$ bond ionisation and migration along the carbon chain. These mass spectra indicated many low mass ions. To prevent this drawback, it was necessary to stabilise double bonds using fatty acid derivatization procedures.

Double bond derivatization was used essentially for mono-unsaturated fatty acids since this technique has limited application to poly-unsaturated fatty acids [29]. One method of double bond derivatization is oxidative ozonolysis. This method resulted in $\pi$ bond cleavage with formation of specific fragments that were different for cis and trans configuration. The fragments were then analysed using GCMS [61].

Methods used mostly in mass spectrometry analysis of unsaturated fatty acids included a derivatization of the carboxylic group into a nitrogenic compound. For instance, most of the trans fatty acid spectrometric analysis are based on GC-MS of either 3-hydroxy-methyl pyridinyl (picolinic esters), or 4,4-dimethyloxazoline (DMOX) derivatives $[4,10,29,40,61]$. These derivatives were fragmented by cleavage of the carbon chain, which resulted in a decrease in low radical ions and an increase of ions corresponding to carbon chain cleavage in methylenic units of 14 atomic mass units. The $\pi$ bond position was determined by breakage of the regular interval of 14 atomic mass units and appearance of a fragment of 12 atomic mass units corresponding to ethylenic residue $[29,62,63]$

Picolinic esters exhibited spectra that were not so easily explained, especially when poly-unsaturated fatty acids and conjugated linoleic acids were analysed [29]. They allowed the determination of fatty acid molecular weight, double bond position on carbon chain, but did not indicate the configuration of these bonds [60]. DMOX spectra were easier to explain since they indicated the double bond position and configuration $[4,29]$. This method is well adapted to polyunsaturated fatty acids and conjugated linoleic acids (Fig. 6).
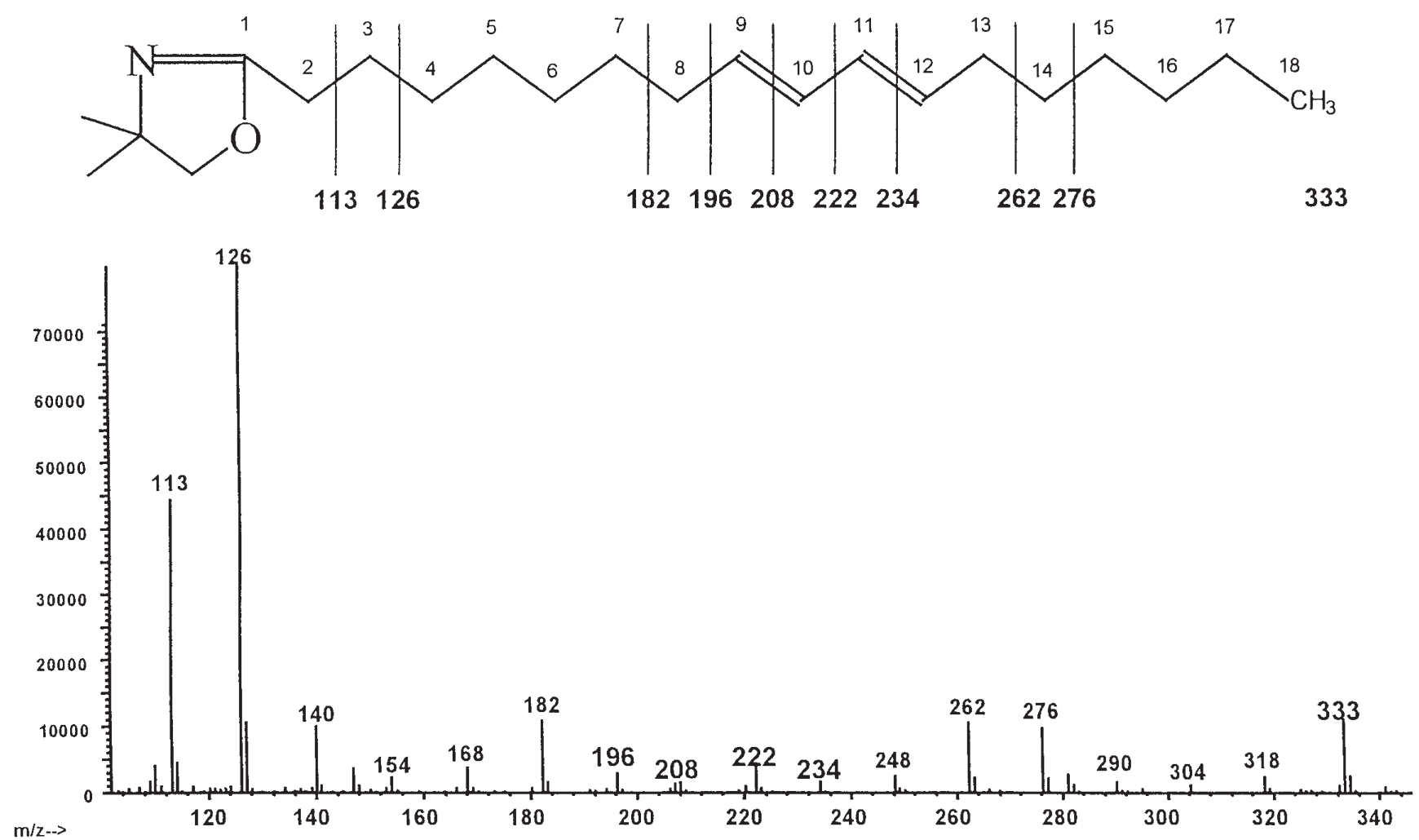

Figure 6. Electron impact mass spectrum of 4,4-dimethyloxazoline (DMOX) derivative of 9-cis, 11-trans 18:2 (rumenic) acid from O. Berdeaux et P. Juaneda, INRA Dijon, UNL (Personal Communication). 
Aro et al. [4] obtained nine peaks for trans-18:1 fatty acid as DMOX derivatives which were quantitated using GC-MS and nine peaks for trans-18:1 fatty acid methyl esters using direct GC with flame ionisation detector. Some peaks included two isomers, but overlapping peaks involved different fatty acids in both methods. The difference in the nature of derivatives brought about a change in the nature of overlapping peaks in the two protocols. So, these authors were able to quantitate up to eleven trans-18:1 isomers by combining these two procedures, by yielding results similar to those obtained using Ag-TLC/GC combination. The authors [4] assumed to replace the laborious and time-consuming Ag-TLC.

\section{Conclusion}

This review examined the major published methods for detection of trans-C18 fatty acid isomers in milk fat. Choice of one method over another depends on the goal of the analysis, in regards to time required or equipment needed.

The use of infrared spectroscopy allowed only a global measure of trans double bonds. Direct GC led to only partial separation of trans and cis isomers. Ag-TLC/GC combination gave individual separation of most positional and geometric isomers that can be quantitated. This method requires equipment that is commonly used in lipid analysis laboratories, but it is time-consuming. Ag-HPLC may be a great improvement of the Ag ion chromatography since it is faster and automation is possible. When associated with gas chromatography-mass spectrometry, Ag ion pre-fractionation methods allowed the identification of each individual isomer.

Knowledge of advantages and drawbacks of each of these techniques will help the analyst to estimate the results published in literature at their true values.

\section{Acknowledgements}

The authors thank Julie A. Nordlee, Department of Food Science and Technology, University of Nebraska-Lincoln, for her valuable assistance in the preparation of the manuscript and P. Chatelain for his help in the preparation of figures.

\section{References}

1. Judd, J.T.; Clevidence, B.A.; Muesing, R.A.; Wittes, J.; Sunkin, M.E.; Podczasy, J.J. Am. J. Clin. Nutr. 1994, 59, 861868.

2. Mensink, R.P.; Katan, M.B. N. Engl. J. Med. 1990, 323, 439445.

3. Mensink, R.P.; Zock, P.L.; Katan, M.B.; Hornstra, G. J. Lipid Res. 1992, 33, 1493-1501.

4. Aro, A.; Kosmeijer-Schuil, T.; van de Bovenkamp, P.; Hulshof, P.; Zock, P.; Katan, M.B. J. Am. Oil Chem. Soc. 1998, 75, 977985.

5. Ascherio, A.; Hennekens, C.H.; Buring, J.E.; Master, C.; Stampfer, M.J.; Willet W.C. Circulation 1994, 89, 94-101.
6. Kris-Etherton, P.M.; Emken, E. A.; Allison, D. B.; Dietschy, J. M.; Nicolosi, R. J.; Denke, M. A. Am. J. Clinic. Nutr. 1995, 62, 655S-707S.

7. Willet, W.C.; Stampfer, M.J.; Manson, J.E.; Colditz, G.A.; Speizer, F.E.; Rosner, B.A.; Sampson, L.A.; Hennekens, C.H. The Lancet 1993, 341, 581-585.

8. Precht, D.; Molkentin, J. Die Nahrung 1995, 39, 343-374.

9. Wolff, R.L. OCL 1994, 3, 209-218.

10. Ratnayake, W.M.N. In: Trans fatty acids in human nutrition; Sébédio, J.L.; Christie, W.W., Eds.; The Oily Press: Ayr, Scotland, 1998; pp 115-161.

11. Tchapla, A. Analusis Magazine 1998, 26, M17-M71.

12. Wolff, R.L.; Precht, D.; Molkentin, J. In: Trans fatty acids in human nutrition; Sébédio, J.L.; Christie, W.W., Eds.; The Oily Press: Ayr, Scotland, 1998; pp 1-33.

13. Wolff, R.L.; Combes, N.A.; Precht, D.; Molkentin, J.; Ratnayake, W.M.N. OCL 1998, 5, 295-300.

14. Emken, E.A. Ann. Rev. Nutr. 1984, 4, 339-376.

15. Precht, D.; Molkentin, J. Narhung 1999, 43, 233-244.

16. Rao, P.C. ; Daubert, B.F. J. Am. Chem. Soc. 1948, 70, 11021104.

17. Deman, L.; Deman, J.M. J. Am. Oil Chem. Soc. 1983, 60, 1095-1098.

18. Lanser, A.C.; Emken, E.A. J. Am. Oil Chem. Soc. 1988, 65 , 1483-1487.

19. Sleeter, R.T.; Matlock, M.G. J. Am. Oil Chem. Soc. 1989, 66, 121-127.

20. Toschi, T.G.; Capella, P.; Holt, C.; Christie, W.W. J. Sci. Food Agric. 1993, 61, 261-266.

21. Ulberth, F.; Haider, H.J. J. Food Sci. 1992, 57, 1444-1447.

22. Ulberth, F.; Henninger, M. J. Am. Oil Chem. Soc. 1992, 69, 829-831.

23. Smith, L.M.; Duncley, W.L.; Franke, A.; Daikiri, T. J. Am. Oil Chem. Soc. 1978, 55, 257-261.

24. Ulberth, F.; Henninger, M. J. Dairy Research 1994, 61, 517527.

25. Ratnayake, W.M.N. J. of AOAC Int. 1995, 78, 783-802.

26. Precht, D.; Molkentin, J. Int. Dairy Journal 1996, 61, 791-809.

27. Kramer, J.K.G.; Felner, V.; Dugan, M.E.R.; Sauer, F.D.; Mossoba, M.M.; Yurawecz, M.P. Lipids, 1997, 32, 1219-1228.

28. Le Quere, J.L.; Semon, E. Analusis Magazine 1998, 26, M40M44.

29. Berdeaux, O.; Juaneda, P.; Sébédio, J.L. Analusis Magazine 1998, 26, M45-M51.

30. Wolff, R.L.; Bayard, C.C. J. Am. Oil Chem. Soc. 1995, 72, 1197-1201.

31. van Vleet, E.S.; Quinn, J.G. J. Chromatogr. 1978, 151, 396400.

32. Sampugna, J.; Pallansch, L.A.; Enig, M.G.; Keeney, M. J Chromatogr. 1982, 249, 245-255.

33. Chen, Z.Y.; Pelletier, G.; Hollywood, R.; Ratnayake, W.M.N. Lipids 1995, 30, 15-21.

34. Thompson, R.H. J. Chromatogr. Sci. 1997, 35, 536-544.

35. Henninger, M.; Ulberth, F. Milchwissenschaft 1994, 49, 555558.

36. Wolff, R.L. J. Am. Oil Chem. Soc. 1994, 71, 277-283,

37. Wolff, R.L.; Precht, D. J. Am. Oil Chem. Soc. 1998, 75, 421422.

38. Ledoux, M. D.E.A. Sciences Alimentaires, Université Orsay Paris XI, 1999. 
39. Precht, D.; Molkentin, J. Fett/Lipid 1997, 99, 319-326.

40. Sehat, N.; Kramer, J.K.G.; Mossoba, M.M.; Yurawecz, M. P.; Roach, J.A.G.; Eulitz, K.; Morehouse, K.M.; Ku, Y. Lipids 1998, 33, 963-971.

41 ISO Norme (projet) ISO/DIS 15304. International Standardization Organization (1998), Genève, Suisse.

42. Kuzdzal-Savoie, S.; Raymond, J. Bioch., Biophys. 1965, 5, 497-511.

43. Wolff, R.L. J. Chromatogr. Sci. 1992, 30, 17-22.

44. Nikolova-Damyanova, B. In: Advances in lipid methodology, Christie, W.W. Ed.; The oily Press: Ayr, 1992; pp 181-237.

45. Wolff, R.L.; Bayard, C.C.; Fabien, R.J. J. Am. Oil Chem. Soc. 1995, 72, 1471-1483.

46. Wolff, R.L. In: New trends in lipid and lipoprotein analysis, Sébédio, J.L.; Perkins, E.G., Eds.; AOCS Press: Champaign, USA, 1995: pp 147-180.

47. Ratnayake, W.M.N.; Pelletier, G. J. Am. Oil Chem. Soc. 1992, 69, 95-105.

48. Wolff, R.L. Lipid Technology 1999, 11, 16-18.

49. Christie, W.W.; Mc G. Breckenridge, G.H. J. Chromatogr. 1989, 469, 261-269.

50. Battaglia, R.; Frohlich, D. Chromatographia 1980, 13, 428431.
51. Christie, W. W. J. High Resolut. Chromatogr. Chromatogr. Commun. 1987, 10, 148-150.]

52. Sehat, N.; Yurawecz, M.P.; Roach, J.A.G.; Mossoba, M.M.; Kramer, J.K.G.; Ku, Y. Lipids 1998, 33 (2), 217-221.

53. Sehat, N.; Rickert, R.; Mossoba, M.M.; Kramer, J.KG.; Yurawecz, M.P.; Roach, J.A.G.; Adlof, R.O.; Morehouse, K.M.; Fritsche, J.;Eulitz, K.; Steinhart, H.; Ku, Y. Lipids 1999 , 34, 407-413.

54. Juaneda, P.; Sébédio, J.L. J. High Resolution Chromatogr. 1994, 17, 321-324.

55. Ulberth, F.; Gabernig, R.G.; Shrammel, F. J. Am. Oil Chem. Soc. 1999, 76 (2), 263-266.

56. Christie, W.W. Lipid Technology 1995, 64-66.

57. Molkentin, J.; Precht, D. Chromatographia 1995, 41, 267-272.

58. Wolff, R.L.; Sébédio, J.L. J. Am. Oil Chem. Soc. 1994, 71, 117-126.

59. Molkentin, J.; Precht, D. Milchwissenschaft 1997, 52, 380-384.

60. Leth, T. Z Lebensm. Unterrs. Forsch. A 1997, 205, 111-115.

61. Sommerfeld, M. Prog. Lipid Res. 1983, 22, 221-233.

62. Anderson, B.A.; Christie, W.W.; Holman, R.T. Lipids 1975, 10, 215-219.

63. Werner, S.A.; Luedecke, O.; Shultz, T.D. J. Agric. Food Chem. 1992, 40, 1817-1821. 